\title{
FACTORES DE RIESGO ASOCIADOS A PROLAPSO GENITAL DE ÓRGANOS PÉLVICOS EN PACIENTES ATENDIDAS EN EL HOSPITAL MILITAR CENTRAL DURANTE EL PERIODO DE ENERO- DICIEMBRE 2018
}

\author{
Claudia F. Valencia-Chión ${ }^{1,4}$, Jhony A. De la Cruz-Vargas ${ }^{2,3}$, Lucy E. Correa-López ${ }^{3}$, Pedro M. Arango-Ochante ${ }^{4,5}$ \\ RESUMEN \\ Objetivo: Determinar cómo los factores de riesgo se asocian a prolapso genital de órganos pélvicos en pacientes atendidas \\ en el Hospital Militar Central. Materiales y métodos: El diseño de investigación fue observacional, cuantitativo, analítico, \\ retrospectivo y, de tipo casos y controles; siendo los casos las mujeres con diagnóstico de prolapso de órganos pélvicos \\ atendidas en el Servicio de Ginecología en el Hospital Militar Central durante el periodo de Enero a Diciembre 2018. \\ Se calcularon los OR con sus respectivos IC95\% con el software estadístico SPSS. Resultados: De las 160 pacientes \\ estudiadas, la media de los casos fue de 65,1 años $\pm 15,1$ y de los controles de 56,2 años $\pm 21,6$. En el análisis multivariado \\ se encontró que el prolapso de órganos genital de órganos pélvicos estuvo asociado con el antecedente de parto vaginal \\ (OR=3.15; IC 1.36-7.31) y la edad mayor de 42 años (OR=6,09; IC 1,65-22,47) y la multiparidad (OR=10.93; IC: 4.27-27.96) \\ Conclusiones: El antecedente de parto vaginal, la edad y la multiparidad son factores de riesgo para que las mujeres \\ presenten prolapso genital de órganos pélvicos.
}

Palabras clave: Prolapso de órgano pélvico; Factor de riesgo; Paridad; Obesidad (Fuente: DeCS BIREME).

\section{RISK FACTORS ASSOCIATED WITH GENITAL PROLAPSE OF PELVIC ORGANS IN PATIENTS ATTENDED IN THE CENTRAL MILITARY HOSPITAL DURING THE PERIOD JANUARY - DECEMBER 2018}

\begin{abstract}
Objective: To determine the risk factors for genital prolapse of pelvic organs in patients treated at the Central Military Hospital. Materials and methods: The research design was observational, quantitative, analytical, retrospective and, of cases and controls type; being the cases the women with diagnosis of pelvic organ prolapse in the Gynecology Service at the Central Military Hospital during the period from January to December 2018. The ORs were found with their respective 95\% $\mathrm{Cl}$ and the statistical evaluation was performed with the statistical software SPSS. Results: Of the 160 patients studied, the average of the cases was 65.1 years \pm 15.1 and of the controls 56.2 years \pm 21.6 . In the multivariate analysis it was found that the prolapse of the genital organs of the pelvic organs was associated with the history of vaginal delivery $(\mathrm{OR}=3.15 ; \mathrm{Cl}$ 1.36-7.31), the age over 42 years $(\mathrm{OR}=6.09 ; \mathrm{Cl} 1,65-22.47)$ and multiparity $(\mathrm{OR}=10.93 ; \mathrm{Cl}$ : $4.27-27.96)$ Conclusions: The history of vaginal delivery, age and multiparity are risk factors for women to have pelvic organ prolapse.
\end{abstract}

Keywords: Pelvic Organ Prolapse; Risk Factors; Parity; Obesity. (Source: MeSH NLM).

\section{INTRODUCCIÓN}

Se conoce como distopia genital al descenso de un órgano pélvico (útero, cúpula vaginal, recto o vejiga) a través del canal vaginal producido por el relajamiento de las estructuras de soporte del suelo pélvico'.

A nivel mundial, el prolapso genital es un problema muy común que tiene una prevalencia estimada entre un 43 a $76 \%$ de la población femenina general².
Se estima que, en Perú el prolapso genital de órganos pélvicos femeninos puede llegar a presentarse hasta en el $33 \%$ de las mujeres, convirtiéndose en una de las principales indicaciones de cirugía ginecología, ya que a su vez el $33 \%$ de estas serán sometidas a una intervención quirúrgica para reparar el defecto ${ }^{3,4}$.

Cuando se clasifica según el tipo de prolapso, el de grado II resulta ser más frecuente, seguido del grado III $(50,4 \%$ vs. $46,7 \%$ ). La edad promedio se considera a los 55 años ${ }^{5}$.

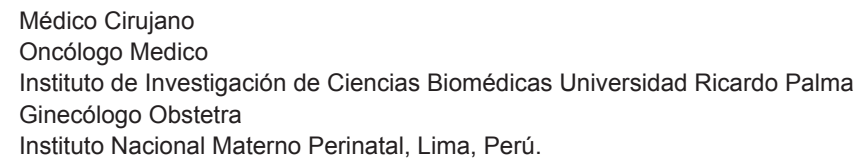

Citar como: Valencia-Chión CF, De La Cruz JA, Correa-López LE, Arango-Ochante PM. Factores de riesgo asociados a prolapso genital de órganos pélvicos en pacientes atendidas en el hospital militar central durante el periodo de enero-diciembre 2018. Rev Peru Investig Matern Perinat 2020; 9(1): 11-6. DOI https://doi.org/10.33421/inmp.2020184 
Sin embargo, diversos autores opinan que se encuentra infradiagnosticado, debido a la inconsistencia de los síntomas, así mismo, porque no es causa de consulta hasta que el prolapso haya superado el plano de la vulva o se halle una masa prominente en la vagina, es por esto que se cree que llega a presentarse hasta en el $50 \%$ de las mujeres multíparas ${ }^{3,6}$.

La etiología de esta patología se considera multifactorial, sin embargo, el principal factor que aumenta el riesgo de padecerla entre 4 a 11 veces más, es el antecedente de parto vaginal ${ }^{7}$.

Por otro lado, se reporta que, a mayor paridad, se correlaciona con tipos más avanzados de prolapso genital; esto se debe a que en un parto prolongado o precipitado no se produzca el relajamiento de las estructuras pélvicas ${ }^{4}$.

Otro factor frecuentemente descrito es la edad ya que el número de cirugías realizadas aumenta a medida que la mujer alcanza la menopausia ${ }^{5}$, llegando a ser el $11 \%$ de cirugías en mujeres de 80 años ${ }^{1}$; así mismo, se estima que el riesgo de tener una intervención por esta causa alcanza el $30 \%$. Es considerada un factor que favorece esta patología debido a que, a mayor edad, se produce la relajación del tono muscular del piso pélvico y la reducción del tamaño del útero ${ }^{4,7}$.

También encontramos otros factores de riesgo modificables como el sobrepeso. el estreñimiento, procesos bronquiales crónicos como el asma, bronquitis crónica o bronquiectasia), levantar cargas pesadas en forma repetitiva (esfuerzos violentos físicos) y no modificables como la raza, el hipoestrogenismo, trauma, colagenopatías, factores genéticos y cirugías previas que hayan producido disrupción del soporte natural ${ }^{5,7}$.

Sin embargo, estos factores de riesgo han sido establecidos en poblaciones distintas a la nuestra, recae ahí la importancia del presente estudio para el reconocimiento de aquellas variables que pueden influir y que aún no han sido exploradas en su totalidad en la población peruana; asi mismo, es muy probable que el impacto del prolapso en la atención de la salud se expanda, debido a que tendencia creciente de este problema se debe al incremento de la longevidad femenina.

\section{MATERIALES Y MÉTODOS}

Tipo y diseño de investigación. El diseño de investigación del presente estudio es de tipo observacional, cuantitativo, analítico, retrospectivo y, de tipo casos y controles.

Población y muestra. Para el cálculo del tamaño muestral se utilizó el calculador brindado por el curso de tesis. Se seleccionó la opción de cálculo de tamaño muestral para estudios de tipo caso-control. Para ello se tomó como nivel de confianza al $95 \%$, una potencial estadística del $80 \%$, razón de controles por caso de 1. El cálculo final de tamaño muestral ajustado con la prueba de Fleiss con corrección de continuidad fue de 160 pacientes, divididos en 80 casos (pacientes con diagnóstico de prolapso genital de órganos pélvicos) y 80 controles. (Pacientes sin el diagnóstico de prolapso genital de órganos pélvicos). Se seleccionó a las pacientes que pertenecerían a los grupos de casos y control según la técnica de muestreo no probabilístico por conveniencia. Se excluyeron las historias clínicas de las pacientes mujeres con gestación actual, cáncer de órgano pélvico, nulíparas y las que no contaban con información completa y confiable.

Variables e instrumentos. La variable dependiente fue el prolapso genital de órganos pélvicos mientras que las variables independientes fueron el antecedente de parto vaginal, la edad, la obesidad, la multiparidad y el tabaquismo.

En el presente estudio se utilizó la información de las historias clínicas de las pacientes con diagnóstico de prolapso genital de órgano pélvico del servicio de Ginecología del Hospital Militar Central durante el periodo enero-diciembre 2018 y que cumplieron con los criterios de selección correspondiente.

Se recogió los datos que proveen los factores de riesgo asociados a prolapso genital de órgano pélvico utilizando una ficha de recolección de datos diseñada por el autor, la cual constó de tres partes: los datos personales, la presencia de prolapso de órgano pélvico y la presencia de los factores de riesgo: edad, antecedente de parto vaginal, obesidad, multiparidad y tabaquismo.

Procesamiento y análisis de datos. Los datos recolectados fueron registrados en una hoja de cálculo de Microsoft Excel, posterior a ello, dicha Hoja fue sometida a un proceso de control de calidad que consistió en seleccionar 5 fichas de recolección de datos al azar y contrastar los datos con los registrados en la Hoja de cálculo para evitar la omisión o el ingreso de datos erróneos. Respecto al plan de análisis, las variables cualitativas serán descritas mediante frecuencias y porcentajes. Las variables cuantitativas serán analizadas según su Normalidad y posteriormente descritas con medidas de tendencia central y dispersión según sea el caso. En el análisis bivariado para determinar las diferencias significativas entre los grupos de categorías, se utilizó en el caso de variables cualitativas pruebas de chi cuadrado o test exacto de Fisher, y para variables cuantitativas las pruebas de $t$ de student o $U$ de Mann Whitney según sea el caso con un Intervalo de Confianza del 95\% y un $p<0.05$ significativo. Posterior a ello, aquellas variables que demostraron diferencias significativas no debidas al azar fueron analizadas mediante el uso de regresiones logísticas utilizando como medida de riesgo el Odss Ratio (OR). El análisis de datos será realizado utilizando 
el programa estadístico SPSS Statidistics con licencia adquirida por el Instituto de Investigación en Ciencias Biomédicas de la Universidad Ricardo Palma.

Aspectos éticos. El presente estudio se cuenta con la autorización del comité de Investigación y Ética del Hospital Militar Central y de la Universidad Ricardo Palma, por ser un estudio de casos y controles, de tipo retrospectivo, en donde solo se recogieron datos clínicos de las historias de los pacientes; no fue necesario el consentimiento informado, así mismo, se tomara en cuenta la declaración de Helsinki II (Numerales: 11,12,14,15,22 y 23) y la ley general de salud (Titulo cuarto: artículos 117 y 120).

\section{RESULTADOS}

La edad promedio de las pacientes del grupo de los casos fue de 65,1 años con un rango de 40 a 90 años, y la edad promedio de las pacientes del grupo control fue de 56,2 años en con un rango de 18 a 92 años.

Los casos presentaron no solo mayor edad promedio que los controles sino también mayor número de partos $(3,5$ en promedio comparado con 1,6 en promedio).

Se observa también que el parto vaginal, la multiparidad, el tabaquismo y la incontinencia urinaria fue más frecuente en el grupo de casos que de controles.

Tabla 1. Características generales de las mujeres que acudieron a los consultorios externos del Servicio de Ginecología en el Hospital Militar Central. EneroDiciembre 2018.

\begin{tabular}{lcccccc}
\hline \multicolumn{1}{c}{ Variables } & \multicolumn{2}{c}{$\begin{array}{c}\text { Casos } \\
(\mathbf{n = 8 0})\end{array}$} & \multicolumn{2}{c}{$\begin{array}{c}\text { Controles } \\
(\mathbf{n}=\mathbf{8 0})\end{array}$} & \multicolumn{2}{c}{$\begin{array}{c}\text { General } \\
(\mathbf{n}=160)\end{array}$} \\
\cline { 2 - 8 } & $\mathbf{n}$ & $\%$ & $\mathbf{n}$ & $\%$ & $\mathbf{n}$ & $\%$ \\
\hline Edad* $^{*}$ & $65,1 \pm 15,1$ & $56,2 \pm 21,6$ & $60,7 \pm 19,1$ \\
\hline Obesidad & 42 & 52.5 & 40 & 50.0 & 82 & 51.3 \\
Tabaquismo & 46 & 57.5 & 38 & 47.5 & 84 & 52.5 \\
\hline $\begin{array}{l}\text { Incontinencia } \\
\text { urinaria }\end{array}$ & 37 & 46.3 & 12 & 15.0 & 49 & 30.6 \\
\hline Multiparidad & 70 & 87.5 & 35 & 43.8 & 105 & 65.6 \\
\hline $\begin{array}{l}\text { Parto vaginal } \\
\text { Cesáreas }\end{array}$ & 58 & 72.5 & 39 & 48.8 & 97 & 60.6 \\
\hline $\begin{array}{l}\text { Número } \\
\text { de partos }\end{array}$ & 22 & 27.5 & 41 & 51.3 & 63 & 39.4 \\
\hline
\end{tabular}

*media y desviación estándar

De las características generales de las pacientes, Tabla 2 , no se halló una asociación entre el prolapso ni con la obesidad $(p=0,752)$ ni con el tabaquismo $(p=0,205)$; y con respecto a la edad, ésta tuvo una asociación fuerte, indicando que las pacientes de edad mayores de 42 años fueron más frecuentes en el grupo de casos $(93,8 \%)$ que en el grupo de controles $(68,8 \%)$, es decir la edad sí es un factor de riesgo asociado al prolapso.
Tabla 2. Análisis bivariado de los factores asociados a prolapso genital en mujeres que acudieron a los consultorios externos del Servicio de Ginecología en el Hospital Militar Central. Enero-Diciembre 2018.

\begin{tabular}{|c|c|c|c|c|c|c|c|c|}
\hline & \multicolumn{2}{|c|}{$\begin{array}{l}\text { Casos } \\
(\mathrm{n}=80)\end{array}$} & \multicolumn{2}{|c|}{$\begin{array}{c}\text { Control } \\
(n=80)\end{array}$} & \multicolumn{2}{|c|}{ Total } & \multirow{2}{*}{ OR } & \multirow{2}{*}{$p$ valor } \\
\hline & $\mathbf{n}$ & $\%$ & $\mathrm{n}$ & $\%$ & $\mathbf{n}$ & $\%$ & & \\
\hline \multicolumn{9}{|c|}{ Edad (años) } \\
\hline 43 a 92 & 75 & 93,8 & 55 & 68,8 & 130 & 81,3 & 6,8 & 0,001 \\
\hline 18 a 42 & 5 & 6,4 & 25 & 31,3 & 30 & 18,8 & & \\
\hline Obesidad & & & & & & , & & \\
\hline Sí & 42 & 52,5 & 40 & 50,0 & 82 & 51,3 & 1,1 & 0,752 \\
\hline No & 38 & 47,5 & 40 & 50,0 & 78 & 48,8 & & \\
\hline \multicolumn{9}{|l|}{ Fuma } \\
\hline Sí & 46 & 57,5 & 38 & 47,5 & 84 & 52,5 & 1,5 & 0,205 \\
\hline No & 34 & 42,5 & 42 & 52,5 & 76 & 47,5 & & \\
\hline
\end{tabular}

Al desglosar la edad en 2 categorías, se encontró que tuvo una asociación muy significativa con el prolapso $(p=0.001)$, pues a medida que aumenta la edad aumenta el riesgo de prolapso, por ejemplo, en las menores de 42 años el $5 \%$ tuvo prolapso y este porcentaje aumentó a $75 \%$ entre las pacientes mayores de 43 años, obteniendo un OR de 6.8 , lo que indica que existe 6,8 veces mayor riesgo de prolapso en aquellas pacientes cuyas edades están entre 43 y 92 años comparado con aquellas pacientes cuyas edades se encuentran entre 18 y 42 años.

En el Gráfico 1 se observa el efecto que tiene la interacción de la edad y la incontinencia urinaria. De los 50 años hasta los 80 indudablemente el prolapso es más frecuente entre las pacientes con incontinencia urinaria (línea roja), pero pasados los 80 años de edad se invierten las tasas de prolapso, es decir, a esa edad la causa de la incontinencia urinaria se debe más a la edad que al prolapso.

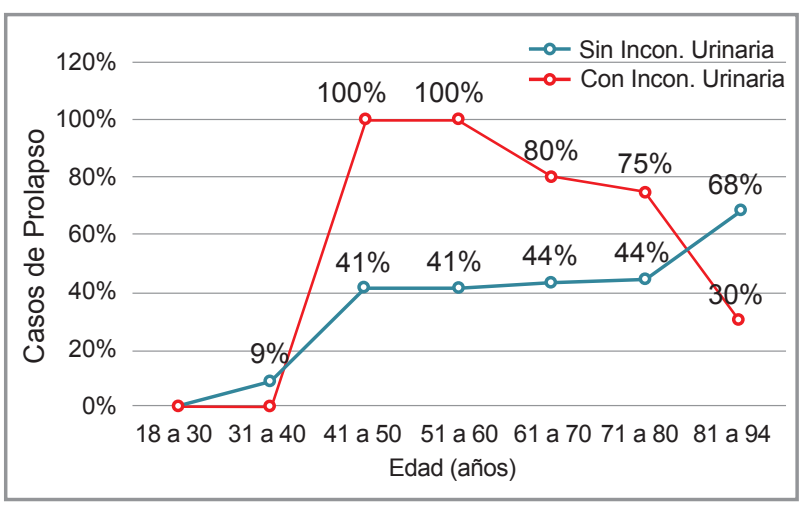

Gráfico 1. Asociación entre la Incontinencia urinaria y el prolapso genital de órganos pélvicos en mujeres que acudieron a los consultorios externos del Servicio de Ginecología en el Hospital Militar Central. Enero-Diciembre 2018. 
Tabla 3. Características gineco-obstétricas en mujeres de edad avanzada que acudieron a los consultorios externos del Servicio de Ginecología en el Hospital Militar Central. Enero-Diciembre 2018.

\begin{tabular}{lrrrrrrr}
\hline \multirow{2}{*}{ Factores } & \multicolumn{2}{c}{ Casos } & \multicolumn{2}{c}{ Controles } & \multirow{2}{*}{$\begin{array}{c}\text { Chi } \\
\text { cuadrado }\end{array}$} & p valor \\
\cline { 2 - 6 } & $\mathbf{n}$ & $\%$ & $\mathbf{n}$ & $\%$ & & \\
\hline Número de partos & & & & & \\
\hline De 1 a 2 & 19 & 23,8 & 68 & 85,0 & & \\
De 3 a 4 & 43 & 53,8 & 11 & 13,8 & 61,771 & $<0,001$ \\
\hline De 5 a 7 & 18 & 22,5 & 1 & 1,3 & & \\
Parto Vaginal & & & & & & \\
\hline Si & 58 & 72,5 & 39 & 48,8 & & \\
\hline No & 22 & 27,5 & 41 & 51,3 & 9,452 & 0,002 \\
\hline
\end{tabular}

De la Tabla 3, se observa que el número de partos resultó un factor fuertemente asociado al prolapso genital $(p<0.001)$, pues más de la mitad de pacientes con prolapso (casos) tuvieron entre 3 a 4 partos (53.8\%), en cambio entre las pacientes sin prolapso (controles) solo el $13.8 \%$ tuvo de 3 a 4 partos.

Esta variable, número de partos, también puede ser expresada como multiparidad y también estuvo asociada con el prolapso, es decir, las multíparas tienen mayor riesgo de prolapso que las primíparas $(p<0.001)$.

El parto vaginal resultó un factor de riesgo asociado a prolapso genital, pues el $72.5 \%$ de pacientes con prolapso presentó parto vaginal, en cambio entre las pacientes sin diagnóstico de prolapso solo el $48.8 \%$ tuvo un parto vaginal, siendo esta diferencia porcentual muy significativa $(p=0.002)$.

Al cruzar la multiparidad con el diagnóstico de prolapso se encontró una asociación muy significativa entre ambas variables $(p<0.001)$. En el grafico 2 , se observa que el $87.5 \%$ de casos de prolapso son multíparas, en cambio entre los controles ese porcentaje se reduce a $12.5 \%$, esto indica que la multiparidad es un factor de riesgo de prolapso genital.

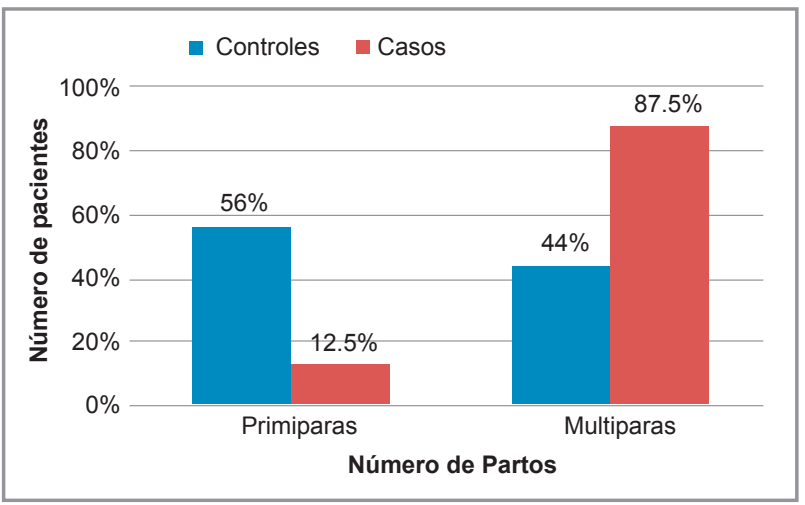

Gráfico 2. Asociación entre el número de partos y el prolapso genital de órganos pélvicos en mujeres que acudieron a los consultorios externos del Servicio de Ginecología en el Hospital Militar Central. Enero-diciembre 2018
Tabla 4. Factores de riesgo según el análisis multivariado de prolapso genital de órganos pélvicos en mujeres que acudieron a los consultorios externos del Servicio de Ginecología en el Hospital Militar Central. EneroDiciembre 2018.

\begin{tabular}{lrc}
\hline \multicolumn{1}{c}{ Factores } & $\begin{array}{c}\text { OR } \\
\text { ajustado }\end{array}$ & IC 95\% \\
\hline Edad & & \\
\hline 43 a 63 & 6,09 & $1,65-22,47$ \\
18 a 42 & 1,00 & \\
Tipo de parto & & \\
$\quad$ Vaginal & 3,15 & $1,36-7,31$ \\
$\quad$ Cesárea & 1,00 & \\
Multípara & & \\
Si & 10,93 & $4,27-27,96$ \\
No & 1,00 & \\
\hline
\end{tabular}

Hasta aquí el en el análisis bivariado, hemos determinado en forma individual qué factores fueron significativos; sin embargo, debemos averiguar cuál es el efecto conjunto o combinado de estos factores sobre el prolapso, por ejemplo tener un parto vaginal con lleva casi 3 veces más riesgo de prolapso que un parto por cesárea $(\mathrm{OR}=2.8$, IC95\%=1.4-5.7), pero este OR ¿no estará siendo influenciado por la edad u otro factor? Para deslindar este asunto se ha aplicado un modelo de regresión logística multivariado.

Edad.Con una confianza del 95\% (IC95\%) se halló que la edad es un factor de riesgo de prolapso, esto es, las pacientes mayores de 42 años tienen 6 veces más riesgo de sufrir un prolapso que las pacientes menores de 42 años $(O R=6,09$, IC95\%=1,65-22,47)

Tipo de parto. Las pacientes con parto vaginal tienen 3 veces más riesgo de prolapso con respecto a las pacientes que tuvieron un parto por cesárea $(\mathrm{OR}=3,15$, IC $95 \%=1,36$ - 7,31). Vemos que el riesgo aumenta ligeramente, pues el parto vaginal sin el efecto de otros factores (edad, incontinencia urinaria, etc.) era de $\mathrm{OR}=2.8$, pero considerando otros factores el riesgo se eleva a $\mathrm{OR}=3.2$, siendo este el riesgo más preciso que el valor individual.

Multiparidad. Este factor resultó muy significativo $(p<0.001)$, y tiene mayor riesgo de prolapso que los otros factores, e indica que el riesgo de prolapso en pacientes multíparas es de 11 veces más en relación a las pacientes primíparas $(\mathrm{OR}=10,93 \quad \mathrm{IC} 95 \%=4.27-27.96)$, en otras palabras, una pacientes primípara tendrá un $91 \%$ más protección contra un prolapso que una multípara.

$\mathrm{Ni}$ el tabaquismo ni la obesidad resultaron ser un factor de riesgo de prolapso $(p>0.05)$ en este trabajo.

Se realizó un subanálisis agrupando a las mujeres en edad avanzada, mayores de 60 , y menores de 60 que se ve reflejado en las tablas 5 y 6 . 
Tabla 5. Factores de riesgo asociados a prolapso en mujeres de edad avanzada que acudieron a los consultorios externos del Servicio de Ginecología en el Hospital Militar Central. Enero-Diciembre 2018.

\begin{tabular}{lcc}
\hline Factor & OR ajustado & IC 95\% \\
\hline Tipo de parto & 3,71 & $1,41-9,76$ \\
Vaginal & 1,00 & \\
Cesárea & & \\
Multípara & 5,16 & $1,89-14,11$ \\
Si & 1,00 & \\
No & \\
\hline
\end{tabular}

Según la Tabla 5, en las pacientes de edad avanzada, mayores de 60 años, solo dos factores son significativos, el tipo de parto y la multiparidad, es decir, el parto vaginal en estas pacientes tiene casi 4 veces más riesgo de prolapso que las pacientes con parto por cesárea. En forma general el riesgo era de $\mathrm{OR}=3.2$ pero en este grupo apenas aumentó a $\mathrm{OR}=3.7$. En el caso del factor multiparidad el riesgo disminuyó a 5.2, casi a la mitad, esto indica que la multiparidad conlleva mayor riesgo de prolapso en las pacientes menores de 60 que en las mayores de 60 años.

Tabla 6. Factores de riesgo asociados a prolapso en mujeres menores de 60 años que acudieron a los consultorios externos del Servicio de Ginecología en el Hospital Militar Central. Enero-Diciembre 2018.

\begin{tabular}{ccccc}
\hline Factor & OR & p & \multicolumn{2}{c}{ IC95\% } \\
\hline Paridad & & & & \\
3 a 4 & 17.9 & $<0.001$ & 4.4 & 72.4 \\
1 a 2 & 1.0 & & & \\
\hline
\end{tabular}

Finalmente, en el grupo de pacientes menores de 60 años, solo la paridad resultó ser un factor de riesgo de prolapso genital. Se observa que las pacientes con 3 o 4 partos tendrán 18 veces mayor riesgo de prolapso que las pacientes con 1 o 2 partos $(\mathrm{OR}=17.9, \mathrm{IC} 95 \%=4.4-72.4)$.

\section{DISCUSIÓN}

En nuestro estudio se ha encontrado algunos factores de riesgo que corroboran lo que otras investigaciones hallaron, y estos factores son la edad de la paciente, pues mujeres mayores de 42 años tienen mayor riesgo de tener un prolapso; otro factor fue la multiparidad, pues su presencia implica un mayor riesgo de prolapso, y esto es de suma importancia porque conocer el efecto de estos factores es la base para llevar a cabo estrategias, terapias, o talleres que concienticen a las mujeres sobre planificación familiar y sobre las disfunciones del piso pélvico, y darles así una mejor calidad de vida. Sin embargo, hubo dos factores que no concuerdan con los resultados de la literatura mundial, y son el tabaquismo y la obesidad, que no resultaron factores de riesgo significativos en este estudio; por lo tanto, es necesario continuar con otras investigaciones para ver si definitivamente no son factores de riesgo de prolapso en la población femenina peruana.

Las disfunciones del piso pélvico tienen muchas causas que elevan el riesgo de prolapso; por ejemplo, están los factores promotores como es el tabaquismo y la obesidad, o factores estimuladores como la multiparidad, o factores descompensadores como la incontinencia urinaria y la edad de la mujer. El hecho de que el tabaquismo y la obesidad no hayan resultado significativos podría deberse a que en este trabajo no se ha considerado el tipo de prolapso ni su magnitud, por ejemplo la Sociedad Internacional de Continencia (ICS) ha desarrollado una clasificación estandarizada para el prolapso (POPQ), que discrimina el tipo de prolapso según su grado; también está la clasificación del Colegio Americano de Obstetricia y Ginecología entre otras clasificaciones, y quizá al considerar el tipo de prolapso o su grado es posible que el tabaquismo o el exceso de peso tengan alguna relación.

La media de los casos fue de 65,1 años $\pm 15,1$ y de los controles de 56,2 años $\pm 21,6$. Se observa que en promedio la edad fue mayor en los casos con respecto a los controles.

El resultado es similar al trabajo de Espitia ${ }^{6}$, quien obtuvo un promedio de edad en los casos de 57,6 $\pm 11,4$ años, y de 54,9 años \pm 13 en los controles observándose también una edad media mayor en los casos comparados a la de los controles siendo muy similar a nuestro estudio.

Se encontró asociación entre el parto vaginal y el prolapso, similar a lo encontrado por Benites $C^{11}$ (Colombia, 2014) quien refiere que el antecedente de parto vaginal aumenta 1.35 veces la aparición de prolapso; o lo encontrado por Rodrigues $A^{9}$ (Brasil) donde encuentra un OR=7,22; (1.8428.27), o lo estudiado por Caffo $\mathrm{R}^{14}$ (Trujillo, 2017) quien reporta que para esta vía de parto un OR de $3.33(p=0.0219$, $(1.148-9.675)$ y lo encontrado por Tafur $\mathrm{J}^{13}$ (Lima 2016).

Con respecto a la variable edad, se encontró asociación con el prolapso, tal como lo encontrado por Kim $\mathrm{CM}^{12}$ (Japon) donde refiere que edades mayores a 70 años incrementan hasta 16 veces mas el riesgo de prolapso, o lo encontrado por Tafur $\mathrm{J}^{13}$ donde encuentra que una edad mayor a 60 años tiene una asociación con OR 8.429 (IC 95\% 4.144 17.144) o Jorge $\mathrm{F}^{15}$ (Huancayo 2016) donde el $80 \%$ de las mujeres evaluadas con prolapso tenían entre 55-69 años.

Se encontró asociación entre la multiparidad y el prolapso; similar a lo encontrado por Espitia de la Hoz (Colombia, $2015)^{6}$ quien encuentra que más del $38 \%$ de su población estudiada poseía más de 6 gestaciones, así mismo, se relaciona a lo encontrado por Benites $\mathrm{C}^{11}$ quien manifiesta que la multiparidad aumentan 1.21 el riesgo de presentar prolapso lo cual se relaciona con nuestros resultados.

En nuestros resultados no hubo evidencia de que la obesidad sea un factor asociado al prolapso $(p=0.752)$, ni en mujeres maduras ni en mujeres de edad avanzada. 
Para entender por qué la obesidad no resultó un factor de riesgo de prolapso es que, según el trabajo de Tafur $\mathrm{J}^{13}$, el grupo de mujeres analizado eran más jóvenes que el grupo de casos de nuestro estudio.

En el presente estudio no se encontró asociación entre el tabaquismo y el prolapso; esto se puede deber a la forma en la que fue medida, una explicación puede estar relacionada con la frecuencia del consumo y la cantidad, y no solamente si lo consume o no, por lo tanto es recomendable medir esta variable en función de su frecuencia de consumo, tal como algunos autores los hacen ${ }^{6}$, donde definen la presencia del tabaquismo cuando la persona tiene un consumo mínimo de un cigarrillo diario desde hace 6 meses antes de la ocurrencia del prolapso, y efectivamente el tabaquismo resultó un factor de riesgo de prolapso bajo tal condición.

Se puede resumir que los únicos factores que la literatura menciona como de riesgo de prolapso genital de órganos pélvicos, y que nuestro estudio no fueron hallados significativos fueron la obesidad y el tabaquismo, y esto debido a las limitaciones del estudio, las historias clínicas no registran la frecuencia y el número de cigarrillos que consume la paciente; y para la variable obesidad, en nuestra muestra, ambos grupos (casos y controles) poseían cifras similares de mujeres con este diagnóstico, por lo cual no fue posible encontrar diferencias significativas

En tal sentido se concluyen que, el antecedente de parto vaginal resultó ser un factor de riesgo de prolapso genital de órganos pélvicos, con un $\mathrm{OR}=3.15$; IC 1.36-7.31. La edad de las pacientes es un factor de riesgo de prolapso genital de órganos pélvicos, es decir, a mayor edad mayor riesgo y viceversa, donde la edad mayor a 42 años presentaba un $\mathrm{OR}=6,09$; IC 1,65-22,47. La obesidad no mostro ser un factor de riesgo de prolapso genital de órganos pélvicos en nuestro estudio. La multiparidad resultó ser un factor de riesgo de prolapso genital de órganos pélvicos, es decir, las primíparas tienen menos riesgo o están más protegidas frente al prolapso genital que las multíparas. (OR=10.93; IC: 4.27-27.96). El tabaquismo no alcanzó significancia para ser un factor de riesgo de prolapso genital de órganos pélvicos en nuestro estudio.

Financiamiento: Autofinanciado.

Conflicto de interés: Los autores declaran no tener algún conflicto de intereses

\section{REFERENCIAS BIBLIOGRÁFICAS}

1. Cohen D. Female pelvic organ prolapse: what you should know. Rev. Med. Clin. Condes - 2013; 24(2) 202-209. Disponible en: https://www.clinicalascondes.cl/Dev_CLC/media/Imagenes/ PDF\%20revista\%20m\%C3\%A9dica/2013/2\%20marzo/4-Dr. Cohen.pdf

2. Peter $H$, Guzmán R. Diagnosis and management of pelvic organ prolapse, present and future. Rev Med Clin
Condes. 2013; 24(2) 210-217. Disponible en: https://www. clinicalascondes.cl/Dev_CLC/media/Imagenes/PDF\%20 revista\%20m\%C3\%A9dica/2013/2\%20marzo/5-Dietz.MD.pdf

3. Instituto Nacional Materno Perinatal. Guías de práctica clínica y de procedimientos en ginecología del departamento de Ginecología del Instituto Nacional Materno Perinatal. Ministerio de salud. 2011.

4. Reátegui R, Acosta S. Importancia de la reducción del prolapso vaginal anterior severo durante el examen clínico y estudio uro dinámico, en el diagnóstico de incontinencia urinaria oculta. Rev Peru Ginecol Obstet. 2013;59(3): 161-166. Disponible en: http://www.scielo.org.pe/scielo.php?script=sci_ arttext\&pid=S2304-51322013000300003\&lng=es.

5. Luna J. Epidemiologia del prolapso genital. Revista colombiana de Obstetricia y Ginecología. DOI: https://doi.org/10.18597/ rcog.1788

6. Franklin J. Espitia de la Hoz. Factores de riesgo asociados con prolapso genital femenino: estudio de casos y controles. Urol Colomb. 2015;24(1):12-18.

7. Gutiérrez C. Características clínicas y factores de riesgo asociados prolapso genital en pacientes atendidas en consulta externa de ginecología del Hospital II Ramón Castilla de Lima, en el periodo 2012 a 2015. [Tesis de Medicina] Univ. Jorge Basafre, Tacna, 2016.

8. Abreu Y, Martinez J, Rodriguez E, Alerm A, Garcia J. Prolapso de órganos pelvios en la mujer. Revisión bibliográfica. Revista Cubana de Medicina Física y Rehabilitación 2016;8(Supl):99110

9. Rodrigues A, Maria de Oliveira L, de Falco K, Del Roy C, Ferreira M, Batista GM, et al. Fatores de risco para o prolapso genital em uma população brasileira. Rev. Bras. Ginecol. Obstet. [Internet]. 2009;31(1): 17-21. Available from: http:// www.scielo.br/ scielo.php?script=sci_arttext\&pid=S0100$72032009000100004 \&$ Ing $=e n$

10. Fritel X, Varnoux N, Zins M, Breart G, Ringa V. Symptomatic pelvic organ prolapse at midlife, quality of life, and risk factors. Rev Obstet Gynecol. 2009;113(3):609-16. Available from: https://www.ncbi.nlm.nih.gov/pubmed/19300324

11. Benites C. Factores de riesgo asociados a prolapso genital total en pacientes atendidas en Hospital Abel Gilbert Pontón entre enero del 2012 a marzo 2014. [Tesis de Medicina] Univ. Católica de Guayaquil, Ecuador, 2014. Available from: http:// repositorio.ucsg.edu.ec/handle/3317/2183

12. Kim CM, Jeon MJ, Chung DJ, Kim SK, Kim JW. Risk factors for pelvic organ prolapse Available from Int J Gynaecol Obstet. 2007;98(3):248-51.

13. Tafur J. Paridad, obesidad y edad avanzada como factores asociados a prolapso genital de órganos pélvicos en pacientes atendidos en el Hospital Central F.A.P. durante el periodo Julio 2016 a Junio 2017. Disponible en: http://repositorio.urp.edu. pe/handle/URP/1258

14. Caffo R. Paridad y tipo de parto como factores de riesgo asociados a prolapso genital en pacientes del servicio de ginecología del hospital regional docente de Trujillo. julio diciembre 2016. Disponible en: http://repositorio.upao.edu.pe/ handle/upaorep/3042

15. Jorge F. Frecuencia del trastorno de piso pélvico en pacientes del hospital Nacional Madre Niño San Bartolomé, JunioSetiembre 2015 Disponible en: http://repositorio.uncp.edu.pe/ handle/UNCP/456

\section{Correspondencia:}

Claudia Valencia chión

Dirección: Calle Cahuide 262 Dpto. 302 Surco

Correo: claudia.valenciachion@hotmail.com

Teléfono: 994405602 